\title{
Molecular characterization of the zinc finger transcription factor, Osterix
}

\author{
MITSUTOKI HATTA $^{1}$, YOSHITAKA YOSHIMURA ${ }^{1}$, YOSHIAKI DEYAMA ${ }^{1}$, \\ AKIYOSHI FUKAMIZU ${ }^{2}$ and KUNIAKI SUZUKI ${ }^{1}$ \\ ${ }^{1}$ Department of Oral Pathobiological Science, Hokkaido University, Sapporo 060-8586; \\ ${ }^{2}$ Center for Tsukuba Advanced Research Alliance, University of Tsukuba, Tsukuba, Ibaraki 305-8857, Japan
}

Received August 17, 2005; Accepted October 3, 2005

\begin{abstract}
Osterix was identified as a transcription factor expressing, in osteoblasts, required for bone formation. However, the molecular mechanisms of the gene regulation by Osterix remain elusive. In this study, we examined the transactivation property of Osterix by using the Gal4 fusion system reporter assay. We identified the transactivation domain of Osterix, which contains high proline and glycine residues and has an activation property in mammalian and yeast cells. The GST-pull down analysis revealed that the basal transcription factor, TF-IIB, but not TBP, binds to the transactivation domain. Furthermore, we found that Osterix interacts with chromatin remodeling factor, Brg-1, through its C-terminal zinc finger domain in vivo and in vitro. These findings suggest that Osterix possesses functional domains which associate with transcription mediated factors and functions as a transcriptional activator in the nucleus.
\end{abstract}

\section{Introduction}

Osteoblasts are cells of mesodermal origin that play a pivotal role in bone formation. The expression of osteoblast-specific genes, such as alkaline phosphatase, osteocalcin, and type 1 collagen, is activated during osteoblast differentiation. In the nucleus, many transcription factors and transcriptional cofactors dynamically interact with each other and regulate their target gene expression in this period (1-4). The runt family transcription factor, Runx $2 / \mathrm{Cbfa} 1$, has previously been shown to be required for osteoblast differentiation (5-7). Furthermore, Nakashima et al (8) reported that the zinc finger transcription factor, Osterix, plays an essential role during osteoblast differentiation, and contributes to gene expression at a later step than Runx2/Cbfa1.

Correspondence to: Dr Kuniaki Suzuki, Department of Oral Pathobiological Science, Hokkaido University, Sapporo 060-8586, Japan E-mail: ksuzuki@den.hokudai.ac.jp

Key words: Osterix, Sp-1/Kruppel-like transcription factor, transactivation, TF-IIB, Brg-1
Osterix contains the unique $\mathrm{N}$-terminal and middle regions, and three zinc finger domains in the $\mathrm{C}$-terminal region. The C-terminal zinc finger region of Osterix shows high homo$\operatorname{logy}$ to the $\mathrm{Sp}-1 /$ Kruppel-like transcription factors. It is assumed that the $\mathrm{N}$-terminal and middle regions possess a transcriptional regulatory property, and that the three-zincfinger domain recognizes the $\mathrm{Sp}$-1-like $\mathrm{G} / \mathrm{C}$-rich sequence (9-12). Although the study on an Osterix null mouse revealed that Osterix is essential for osteoblast differentiation, the molecular mechanisms controlling osteoblast-specific gene expression by Osterix are still unknown.

In the present study, we first identified the transactivation domain of Osterix by means of the Gal4 fusion system in mammalian cell lines and yeast cells, and showed that the transactivation domain associates with TF-IIB, but not TBP. Furthermore we revealed that the $\mathrm{C}$-terminal zinc finger domain of Osterix binds to chromatin remodeling factor Brg-1. These results indicated that Osterix functions through its multiple domains associating with gene expressionmediated factors in the nucleus.

\section{Materials and methods}

Plasmid construction. Mouse Osterix cDNA was cloned from total cellular RNA of MC3T3-E1 cells by reverse transcription PCR with the oligonucleotide primers, 5'-CGCGGATCCAT GGCGTCCTCTCTGCTTGAGGAA-3' for the 5' side and 5'CGCGGATCCTCAGATCTCTAGCAGGTTGCTCTG-3' for the 3 ' side. pcDNA3-FLAG-Osterix was constructed by subcloning full-length Osterix cDNA in-flame into the BamHI site in pcDNA3-FLAG. GST fusion constructs of full-length Osterix and various deletion mutants were obtained by ligating the appropriate coding sequence downstream and in-frame into pGEX-6P-2 (Amersham Bioscience). pGAL4 control vector was generated by inserting the GAL4 DNA-binding domain [GAL4DBD (amino acid 1-147)] into the HindIII site in pcDNA3 (13). GAL4 fusion constructs of full-length Osterix and various deletion mutants were obtained by ligating the appropriate coding sequence downstream and in-frame into GAL4DBD in pGAL4. pG5b-Luc luciferase reporter plasmid was made by inserting 5 copies of GAL4 binding sequence and TATA sequence in adenovirus E1B promoter upstream into the luciferase coding sequence (13). pRL-CMV control plasmid was obtained from Promega. Yeast expression 
A

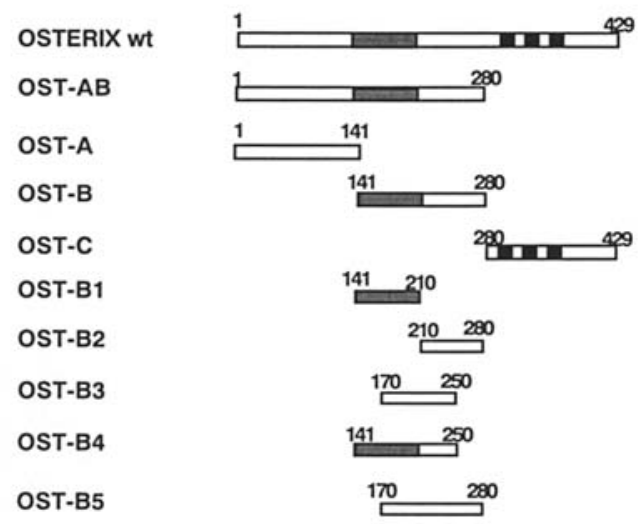

B

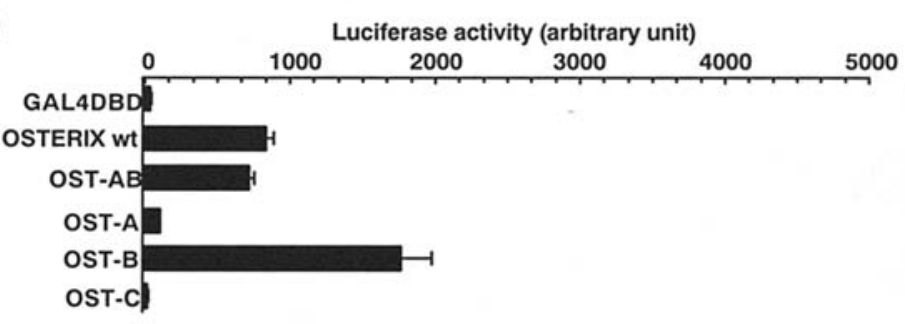

C

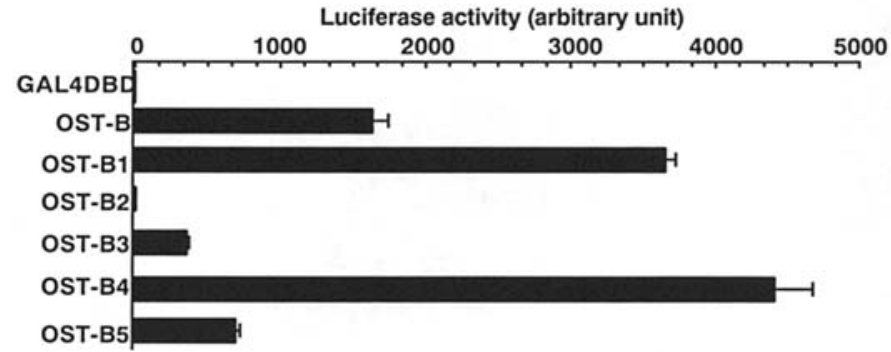

Figure 1. Mapping of the transactivation domain in Osterix. (A) Schematic representations of the structure of Gal4-fused full-length and deletion forms of Osterix used in luciferase assay. (B and C) MG63 cells were plated in 24-well dishes and transfected with 15 ng of G5b-luc, 15 ng of pRL-CMV internal control plasmid, and $15 \mathrm{ng}$ of the Gal4 chimeric genes indicated in (A). Luciferase activity was normalized to Renilla luciferase activity and expressed as the mean of triplicate samples.

GAL4 fusion constructs of full-length Osterix and various deletion mutants were generated by ligating the appropriate coding sequence downstream and in-frame into GAL4DBD in pGBK-T7 (Clontech). pcDNA3-HA-Brg-1 was constructed by inserting full-length human Brg-1 cDNA (a gift from $\mathrm{H}$. Kato) into pcDNA3-HA.

Cell culture. MG63 (osteosarcoma) and HeLa cells (epithelial carcinoma) were obtained from Cell Resource Center for Biomedical Research, Tohoku University (Sendai, Japan). HepG2 (hepatocarcinoma), HeLa, and HEK293T cells (human embryonic kidney) were cultured in Dulbecco's modified minimum essential medium (DMEM) supplemented with $10 \%$ fetal bovine serum, and MG63 cells were grown in MEM supplemented with $10 \%$ fetal bovine serum. All cells were cultured in a $95 \%$ air $/ 5 \% \mathrm{CO}_{2}$ atmosphere, in $95 \%$ humidity, at $37^{\circ} \mathrm{C}$.

Transfection and transactivation assay. Mammalian cells were plated in 24-well dishes and transfected by Fugene-6 reagents (Roche Applied Science) with pG5b-Luc, pGAL4 Osterix expression plasmids, and pRL-CMV at $60 \%$ confluence. pRL-CMV control plasmid was included in each transfection experiment to control for the efficiency of transfection. After transfection, the cells were cultured in DMEM or MEM supplemented with $10 \%$ fetal bovine serum for $48 \mathrm{~h}$, and then harvested using $100 \mu \mathrm{l}$ of passive lysis buffer. Luciferase assay was performed using the dual luciferase reporter assay system (Promega), and samples were loaded on Luminescencer-PSN (ATTO) to measure the luciferase activity.

Saccharomyces cerevisiae strain Y190 was transformed with Osterix deletion mutant expression plasmids by lithium acetate method, and transformants were selected by incubation on agar plates lacking tryptophan for 3 days. The liquid $\beta-$ galactosidase assay was conducted by standard method.

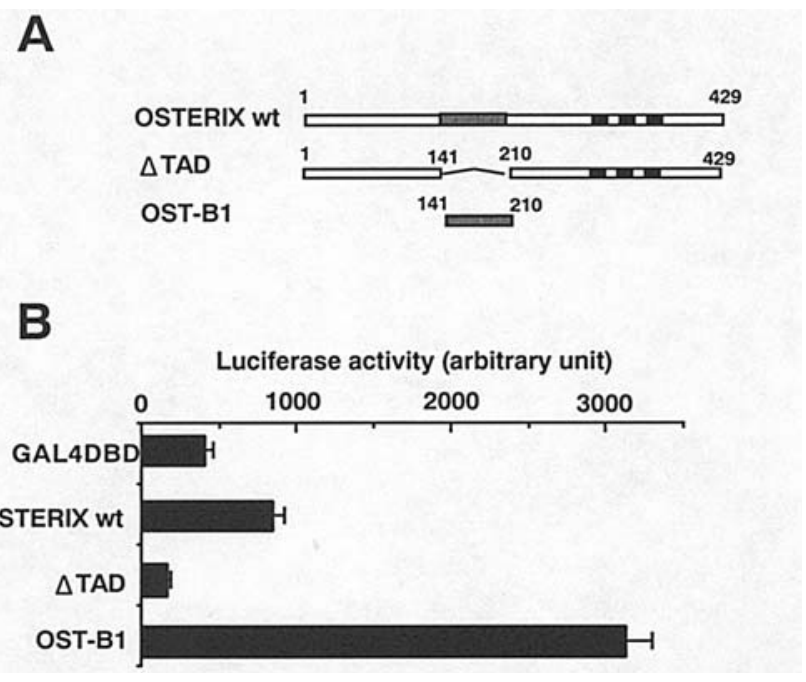

Figure 2. OST-B1 region is essential for transcriptional activity of Osterix. (A) Schematic representations of the structure of Gal4-fused full-length and deletion forms of Osterix used in luciferase assay. (B) MG63 cells were plated in 24-well dishes and transfected with $15 \mathrm{ng}$ of G5b-Luc, $15 \mathrm{ng}$ of pRL-CMV internal control plasmid, and $15 \mathrm{ng}$ of the Gal4 chimeric genes indicated in (A). Luciferase activity was normalized to Renilla luciferase activity and expressed as the mean of triplicate samples.

Briefly, $1 \mathrm{ml}$ of growth medium was inoculated with selected colonies. The cells were harvested and incubated with $\beta$ galactosidase assay buffer containing ortho-nitro-phenolgalactosidase at $30^{\circ} \mathrm{C}$. The activity was measured by an optical density of $420 \mathrm{~nm}$ divided by time of incubation and number of cells.

Antibodies. Mouse monoclonal antibody against the Flag tag (M2) was purchased from Sigma. Rabbit polyclonal antibody against Brg-1 (H-88), rabbit polyclonal antibody against TFIIB (C-18), and mouse monoclonal antibody against TBP 
A

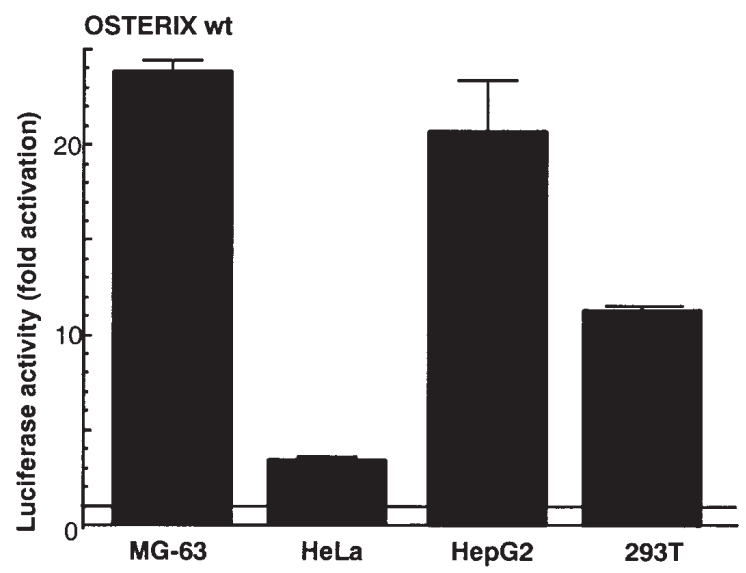

B

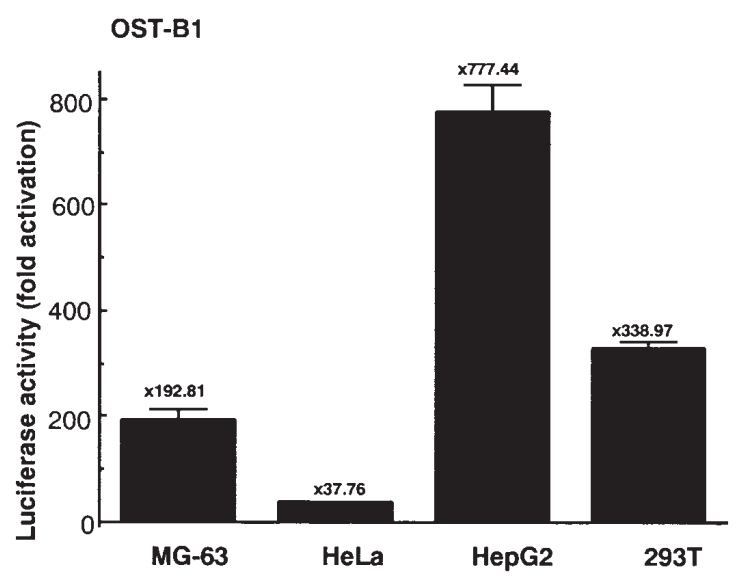

Figure 3. Transcriptional activity of Gal4-fused Osterix in various mammalian cell lines. (A and B) Four cell lines (MG63, HeLa, HepG2, and 293T cells) were plated in 24-well dishes and transfected with $15 \mathrm{ng}$ of G5b-Luc, $15 \mathrm{ng}$ of pRL-CMV internal control plasmid, and $15 \mathrm{ng}$ of the Gal4 chimeric genes. Luciferase activity was normalized to Renilla luciferase activity and expressed as the mean of triplicate samples. Yeast Y190 cells were transformed with the Gal4 chimeric genes indicated in (A). ß-galactosidase activity was expressed as the mean of triplicate samples.

(58C9) were obtained from Santa Cruz Biotechnology. Mouse monoclonal antibody against the HA tag (12CA5) was purchased from Roche Applied Science.

Immunoprecipitation and immunoblotting analysis. HEK293T cells were transfected with pcDNA3 or pcDNA3-FLAGOsterix, and lysed in lysis buffer A [10 mM HEPES-KOH (pH 7.6), $150 \mathrm{mM} \mathrm{KCl,} \mathrm{0.1 \%} \mathrm{NP-40,} 1$ mM PMSF]. The cell lysates were incubated with anti-FLAG antibody (M2) and protein $\mathrm{G}$ sepharose for $2 \mathrm{~h}$ at $4^{\circ} \mathrm{C}$. After washing with lysis buffer A, the immunocomplexes were resolved by SDSpolyacrylamide gel electrophoresis, followed by electrotransfer onto PVDF membrane (Millipore). After transfer, the membrane was incubated with TBS-T (TBS with $0.1 \%$ Tween-20) with 5\% skim milk for $1 \mathrm{~h}$ at room temperature, followed by anti-FLAG antibody (M2) or anti-Brg-1 antibody, overnight at $4^{\circ} \mathrm{C}$. The membrane was washed with TBS-T and incubated with horseradish peroxidase (HRP)-conjugated secondary antibodies for $1 \mathrm{~h}$ at room temperature. Finally, the membrane was washed with TBS-T and detection was conducted using ECL plus (Amersham Biosciences).

GST-pull down analysis. The GST fusion proteins of osterix and osterix deletion mutants were expressed in Escherichia coli strain, BL21, and purified using glutathione-sepharose beads (Amersham Biosciences). The cell extract obtained from HEK293T cells was incubated with $2 \mu \mathrm{g}$ of each GST fusion protein bound to resin for $4 \mathrm{~h}$ at $4^{\circ} \mathrm{C}$. After washing with lysis buffer A, bound proteins were fractionated by SDS-polyacrylamide gel electrophoresis and analyzed by immunoblotting.

\section{Results}

To assess the transactivation activity of Osterix, we utilized the Gal4 fusion system in human osteosarcoma MG63 cells. We constructed the expression plasmids containing full-length Osterix and various deletion fragments in frame to the Gal4 DNA-binding domain (Fig. 1A). As shown in Fig. 1B, the constructs containing the middle region of Osterix (residues 141-280) showed transactivation activity, whereas the Nterminal (residues 1-141) and C-terminal (residues 280-429) regions had no activity. Further analysis using deletion mutants of the middle region revealed that the OST-B1 region (residues 141-210) had strong transactivation activity (Fig. 1C).

To determine whether the OST-B1 region is essential for the transactivation property of Osterix, we constructed the Gal4 fusion $\triangle T A D$ Osterix in which the OST-B1 region is deleted (Fig. 2A), and conducted the reporter assay. $\triangle \mathrm{TAD}$ displayed a significant decrease in the transactivation activity compared with wild-type Osterix and OST-B1 region (Fig. 2B).

Osterix is an osteoblast-specific transcription factor, and essential for bone formation (8). Therefore, we tested whether Osterix can act as a transcriptional activator in various cell lines, which are derived from different tissue. Four cell lines (MG63, HeLa, HepG2, and HEK293T cells) were transfected with G5b-Luc and the Gal4 fusion Osterix, or OST-B1 expression plasmid. Both Osterix (Fig. 3A) and the OST-B1 region (Fig. 3B) provided transcriptional activation in a cell type-independent manner. These results suggest that the OST-B1 region is essential for the transactivation property of Osterix and can act as a transactivation domain in various cell types.

The OST-B1 region contains a unique proline-glycine-rich sequence, which shares no significant similarity with other Sp-1/Kruppel-like transcription factors (Fig. 4A). A previous study reported that the proline-rich activation domain of NF-1/ CTF1 associates with TF-IIB, but not TATA binding protein (TBP), and functions in both mammalian and yeast systems (13). Thus, we examined whether the transactivation domain of Osterix possesses these activities. First, we performed GST-pull down assay, and revealed that the OST-B1 region interacts with TF-IIB, but not TBP. Furthermore, we conducted ß-galactosidase assay by using Y190 yeast cells. As shown in Fig. 4C, $\triangle \mathrm{TAD}$ showed a significant decrease in transactivation activity and the OST-B1 region had transactivation activity in the yeast cells. These results indicate that the 


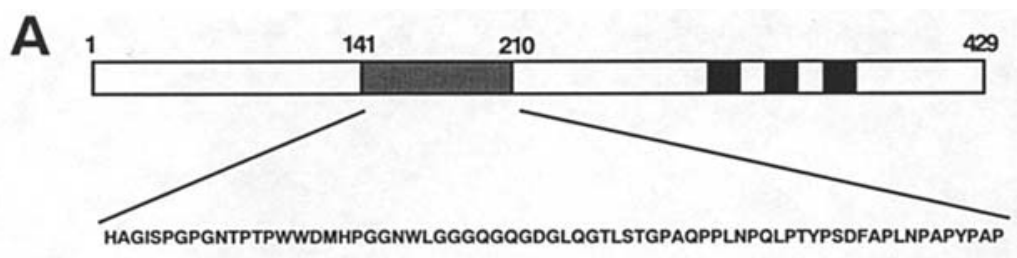

transactivation domain
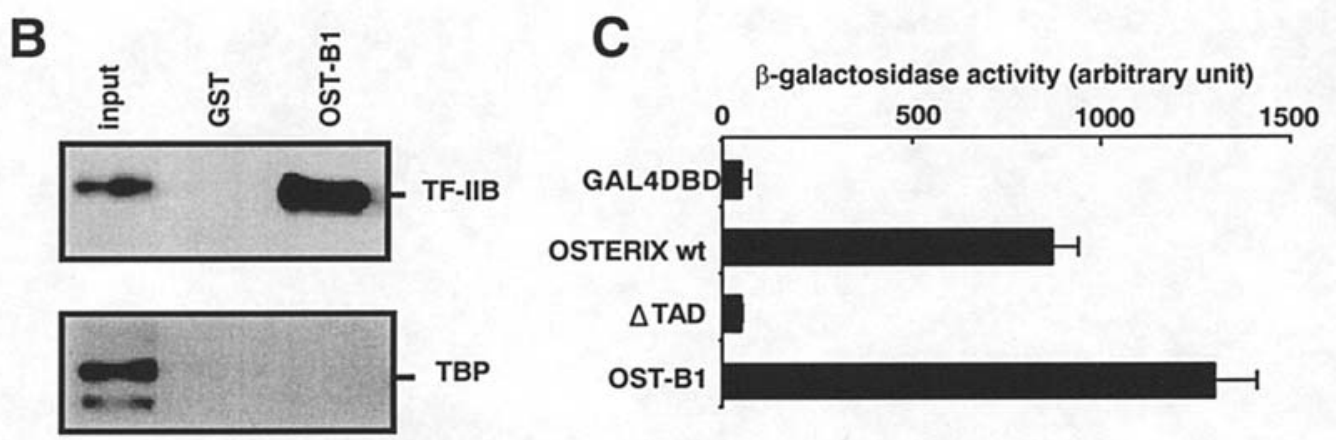

Figure 4. The transactivaton domain of Osterix associates with TF-IIB, but not TBP. (A) Schematic representations of the structure of Osterix and amino-acid sequence of the transactivation domain. (B) Cell extracts from HEK293T cells were incubated with recombinant protein GST, or GST-OST-B1 for $4 \mathrm{~h}$ at $4{ }^{\circ} \mathrm{C}$. After washing with lysis buffer A, binding proteins were detected by immunoblotting with anti-TF-IIB or anti-TBP antibody. (C) Yeast Y190 cells were transformed with the Gal4 chimeric genes indicated in Fig. 2A. B-galactosidase activity was expressed as the mean of triplicate samples.
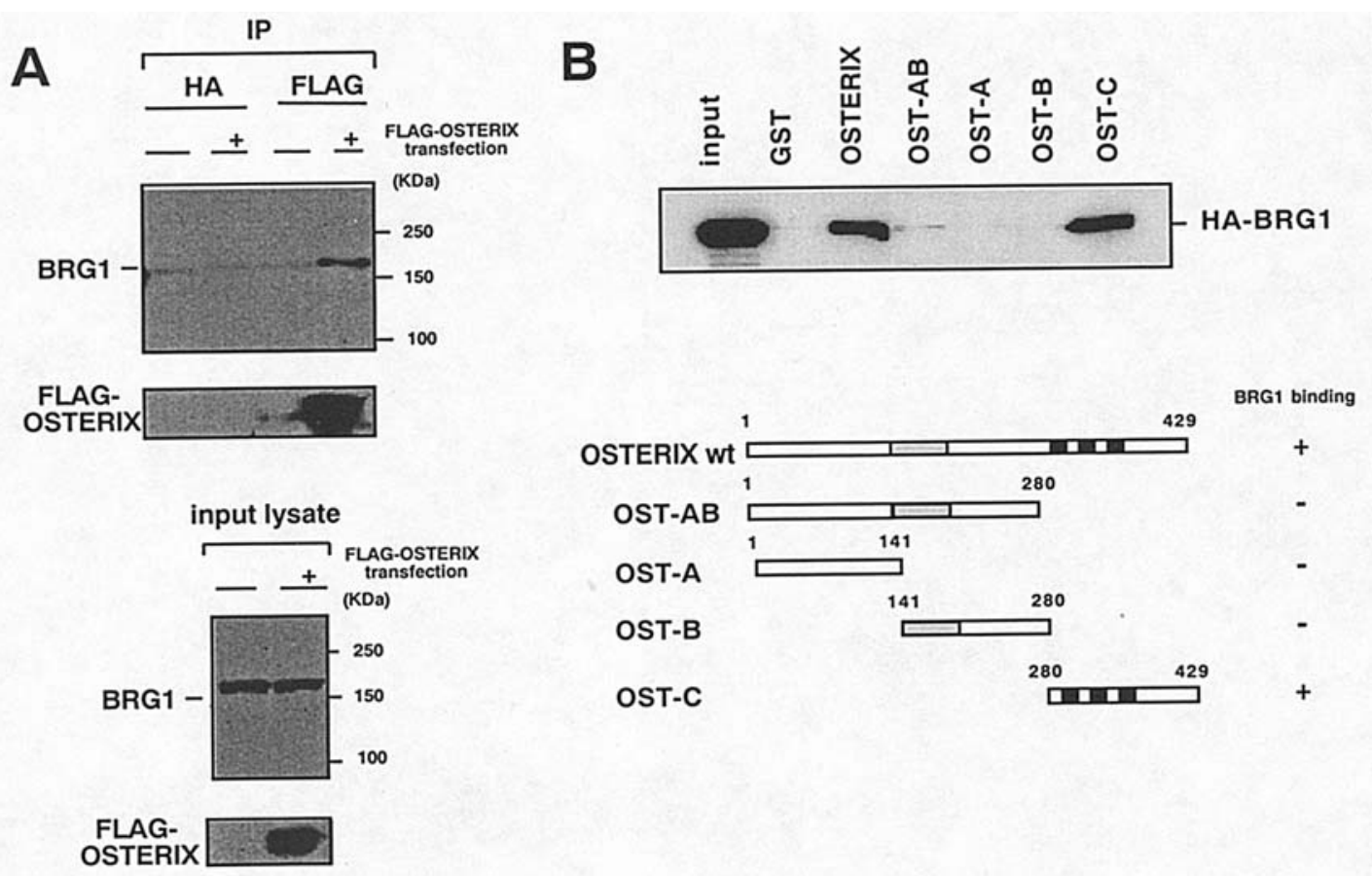

Figure 5. C-terminal zinc finger domain in Osterix associates with Brg-1. (A) Cell extracts from HEK293T cells transfected with pcDNA3-FLAG-Osterix were incubated with anti-FLAG (M2), or anti-HA (12CA5) for $4 \mathrm{~h}$ at $4^{\circ} \mathrm{C}$. After washing with lysis buffer A, binding proteins were detected by immunoblotting with anti-Brg-1 (Santa Cruz), or anti-FLAG (M2) antibody. (B) Upper panel, cell extracts from HEK293T cells transfected with pcDNA3-HA-Brg-1 were incubated with recombinant protein GST, GST-Osterix deletion mutants for $4 \mathrm{~h}$ at $4^{\circ} \mathrm{C}$. After washing with lysis buffer A, binding proteins were detected by immunoblotting with anti-HA (12CA5) antibody. Lower panel, schematic representations of the structure of GST-fused deletion forms of Osterix and binding ability to Brg-1.

proline-glycine-rich OST-B1 region of Osterix, like the proline-rich activation domain of NF-1/CTF1, possesses binding activity to TF-IIB and functions as a transactivation domain in yeast cells.
Tissue-specific transcription factors regulate tissuerestricted gene expression though interaction with transcriptional coactivators, chromatin remodeling complexes, and basal transcriptional machinery (14). EKLF, an erythroid- 


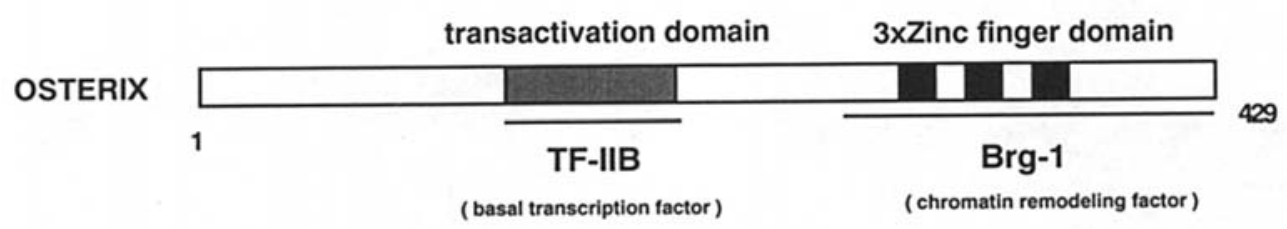

Figure 6. A schematic representation of the functional domain in Osterix. A schematic representation of Osterix. A shaded box indicates the transactivation domain. Closed boxes indicate $\mathrm{C} 2 \mathrm{H} 2$ zinc finger domains. The transactivation domain interacts with TF-IIB, and the C-terminal region binds to Brg-1.

specific Kruppel-like transcription factor, plays an essential role in B-globin gene expression (15). EKLF modulates the chromatin structure of the B-globin gene through the binding activity of the $\mathrm{C}$-terminal zinc finger domain to Brg-1 containing SWI/SNF chromatin remodeling complexes (16). To assess whether Osterix interacts with Brg-1, we first conducted immunoprecipitation analysis, and revealed that Osterix associates with Brg-1 in vivo. To further investigate the binding domain of Osterix to Brg-1 (Fig. 5A), we performed GST-pull down assay by using various Osterix deletion proteins. As shown in Fig. 5B, Brg-1 associated with the C-terminal region of Osterix. These results indicate that the C-terminal domain containing three $\mathrm{C} 2 \mathrm{H} 2$ zinc fingers of Osterix, like EKLF, possesses binding activity to Brg-1.

\section{Discussion}

In this study, we found the functional domains of Osterix possessing binding activity to gene expression-mediated factors (Fig. 6). First, we analyzed the transactivation property of Osterix, and identified the transcriptional activation domain (residues 141-210) containing a proline-glycine rich sequence. The transactivation domains were studied by classifying into the acidic activation domain, the glutamine-rich activation domain, and the proline-rich activation domain. The acidic activation domain was present in yeast transcription factor, GAL4, and herpes simplex virus, VP16. The glutamine-rich activation domain was found in $\mathrm{Sp}-1$, and the proline-rich activation domain was found in NF-1/CTF1. We revealed that the transactivation domain of Osterix, like the prolinerich activator domain of NF-1/CTF1, interacts with TF-IIB, but not TBP, and functions in both mammalian and yeast cells.

Osterix contains three $\mathrm{C} 2 \mathrm{H} 2$ zinc fingers in the C-terminal domain that share a high degree of identity with motifs of $\mathrm{Sp}-1$ and Sp-3. However, the N-terminal and middle regions share no significant similarity with any other transcription factors (8). Furthermore, the transactivation domain of Osterix contains a proline-glycine-rich sequence. On the other hand, Sp-1 and Sp-3 possess the glutamine-rich activation domain. The difference in activation domains may lead to the difference in their roles in gene regulation, since no other $\mathrm{C} 2 \mathrm{H} 2$ zinc finger containing transcription factors, such as Sp-1 and Sp-3, can substitute for the function of Osterix in osteoblast differentiation. The promoter region in the alkaline phosphatase gene contains the $\mathrm{Sp}$-1-like G/C-rich sequence. Several studies reported that $\mathrm{Sp}$ family transcription factors regulate alkaline phosphatase promoter activity $(17,18)$. Thus, we tested whether Osterix potentiates alkaline phosphatase promoter activity. Interestingly, the reporter assay revealed that there is no involvement of Osterix in the regulation of alkaline phosphatase promoter activity (data not shown).

Furthermore, we identified that Osterix interacts with Brg-1. Brg-1 is a catalytic component of SWI/SNF chromatin remodeling complexes. SWI/SNF complexes have been implicated in remodeling local chromatin structure, permitting increased access of transcriptional activators to their recognition sites in the target genes (19). Several studies reported that the transcription factors, such as EKLF, C/EBP, and MyoD, which play an essential role in tissue-differentiation associate with SWI/SNF complexes and regulate the expression of tissue-specific genes $(15,20,21)$. The C-terminal zinc finger of Osterix shares high similarity with EKLF. Thus, it is possible that Osterix targets SWI/SNF complexes to osteoblast specific genes, and regulates osteoblast differentiation. However, less is known about the genes regulated by Osterix in osteoblast. Further study will be needed to assess the functional relevance between osteoblast-specific gene expression and the OsterixBrg-1 interaction.

\section{Acknowledgements}

We thank H. Kato for the human Brg-1 construct, and all members of the Fukamizu Laboratory and Department of Oral Pathobiological Science for various suggestions, discussions, and reagents. This work was supported by a grant from the Northern Advancement Center for Science \& Technology.

\section{References}

1. Ducy P and Karsenty G: Two distinct osteoblast-specific cisacting elements control expression of a mouse osteocalcin gene. Mol Cell Biol 15: 1858-1869, 1995.

2. Karsenty $G$ and De Cronbrugghe B: Conversation of binding sites for regulatory factors in the coordinately expressed alpha 1 (I) and alpha 2 (I) collagen promoters. Biochem Biophys Res Commun 177: 538-544, 1991 .

3. Hatta M, Daitoku H, Matsuzaki M, Deyama Y, Yoshimura Y, Suzuki K, Matsumoto A and Fukamizu A: Regulation of alkaline phosphatase promoter activity by forkhead transcription factor FKHR. Int J Mol Med 9: 147-152, 2002.

4. Funato N, Ohtani K, Ohyama K, Kuroda T and Nakamura M: Common regulation of growth arrest and differentiation of osteoblasts by helix-loop-helix factors. Mol Cell Biol 21: 7416-7428, 2001.

5. Komori T, Yagi H, Nomura S, Yamaguchi A, Sasaki K, Deguchi K, Shimizu Y, Bronson RT, Gao YH, Inada M, Sato M, Okamoto R, Kitamura Y, Yoshiki S and Kishimoto T: Targeted disruption of Cbfa1 results in a complete lack of bone formation owing to maturational arrest of osteoblasts. Cell 89: 755-764, 1997.

6. Ducy P, Zhang R, Geoffroy V, Ridall AL and Karsenty G: Osf2/ Cbfa1: a transcriptional activator of osteoblast differentiation. Cell 89: 747-754, 1997. 
7. Otto F, Thornell AP, Crompton T, Denzel A, Gilmour KC, Rosewell IR, Stamp GW, Beddington RS, Mundlos S, Olsen BR, Selby PB and Owen MJ: Cbfa1, a candidate gene for cleidocranial dysplasia syndrome, is essential for osteoblast differentiation and bone development. Cell 89: 765-771, 1997.

8. Nakashima K, Zhou X, Kunkel G, Zhang Z, Deng JM, Behringer RR and De Crombrugghe $B$ : The novel zinc fingercontaining transcription factor Osterix is required for osteoblast differentiation and bone formation. Cell 108: 17-28, 2002.

9. Philipsen S and Suske G: A tale of three fingers: the family of mammalian Sp/XKLF transcription factors. Nucleic Acids Res 27: 2991-3000, 1999.

10. Suske G: The Sp-family of transcription factors. Gene 238: 291-300, 1999.

11. Turner J and Crossley M: Mammalian Kruppel-like transcription factors: more than just a pretty finger. Trends Biochem Sci 24: 236-240, 1999.

12. Bouwman P and Philipsen S: Regulation of the activity of Sp1related transcription factors. Mol Cell Endocrinol 195: 1-27, 2002.

13. Kim TK and Roeder RG: Proline-rich activator CTF1 targets the TFIIB assembly step during transcriptional activation. Proc Natl Acad Sci USA 91: 4170-4174, 1994.

14. Lemon B and Tjian R: Orchestrated response: a symphony of transcription factors for gene control. Genes Dev 14: 2551-2569, 2000.
15. Nuez B, Michalovich D, Bygrave A, Ploemacher R and Grosveld F: Defective haematopoiesis in fetal liver resulting from inactivation of the EKLF gene. Nature 375: 316-318, 1995.

16. Brown RC, Pattison S, Ree J, Coghill E, Perkins A, Jane SM and Cunningham JM: Distinct domains of erythroid Kruppellike factor modulate chromatin remodeling and transactivation at the endogenous B-globin gene promoter. Mol Cell Biol 22: 161-170, 2002.

17. Terao M, Studer M, Gianni M and Garattini E: Isolation and charactarization of the mouse liver/bone/kidney-type alkaline phosphatase gene. Biochem J 268: 641-648, 1990.

18. Yusa N, Watanabe K, Yoshida S, Shirafuji N, Shimomura S, Tani K, Asano S and Sato N: Transcription factor Sp3 activates the liver/bone/kidney-type alkaline phosphatase promoter in hematopoietic cells. J Leukoc Biol 68: 772-777, 2000.

19. Peterson CL and Workman JL: Promoter targeting and chromatin remodeling by the SWI/SNF complex. Curr Opin Gene Dev 10: 187-192, 2000.

20. Serana IL, Carlson KA and Imbalzano AN: Mammalian SWL/ SNF complexes promote MyoD-mediated muscle differentiation. Nat Genet 27: 187-190, 2001.

21. Pedersen TA, Kowenz-Leutz E, Leutz A and Nerlov C: Cooperation between C/EBP $\alpha$ TBP/TFIIB and SWI/SNF recruiting domains is required for adipocyte differentiation. Genes Dev 15: 3208-3216, 2001. 